\title{
Buscando la salida del laberinto: análisis de la definición de educación para el desarrollo
}

\author{
Noelia Santamaría-Cárdaba ${ }^{1}$ \\ ORCID: 0000-0001-6864-9330
}

\section{Resumen}

La Educación para el Desarrollo es una cuestión clave para lograr una transformación social; por este motivo, esta temática está cobrando especial relevancia en los últimos años al impulsar la formación de personas globales y críticas que promuevan un mundo más humano y sostenible. Esta investigación busca establecer una definición de Educación para el Desarrollo, pues es una cuestión de especial interés debido al dinamismo que posee este término y a la inexistencia de una definición ampliamente aceptada. Por medio de la metodología cualitativa y con ayuda del software de análisis cualitativo NVivo 10 se han analizado 55 definiciones recopiladas en estudios publicados de las bases de datos más relevantes del área de las Ciencias Sociales. Los resultados muestran las relaciones existentes entre los conceptos clave del concepto estudiado: educación y desarrollo, pues permiten vislumbrar las relaciones que emergen de cada uno de estos términos; asimismo, en esta exploración cualitativa se aprecian los rasgos más relevantes de todas las definiciones analizadas, lo cual permite construir un acercamiento fundamentado al concepto de Educación para el Desarrollo. En conclusión, la Educación para el Desarrollo es un proceso educativo que tiene como finalidad promover la formación de ciudadanos globales y críticos que traten de transformar el mundo en un lugar más justo y defiendan los Derechos Humanos.

\section{Palabras clave}

Educación para el Desarrollo - Educación para la ciudadanía - Educación global - Ciudadanía.

1- Universidad de Valladolid, Segovia, España. Contacto: noelia.santamaria.cardaba@uva.es. 


\title{
Looking for the way out of the labyrinth: analysis of the definition of development education
}

\begin{abstract}
Development Education is a key issue for achieving social transformation; that is why this matter has gained special prominence in recent years by encouraging the formation of global and critical people who promote a more humane and sustainable world. This research seeks to establish a definition of Development Education, as it is a subject of special interest due to the dynamism of this term and the lack of a widely accepted definition. By means of the qualitative methodology and with the help of the NVivo 10 qualitative analysis software, 55 definitions compiled in published studies of the most outstanding databases in the area of Social Science were analyzed. The results show the existing relationship between the key concepts involved: education and development, since they allow us to take a glimpse of the relations that emerge from each of these terms; likewise, in this qualitative exploration, the most significant features of all the definitions analyzed are appreciated, which allows us to construct a well-founded approach to the concept of Development Education. In conclusion, Development Education is an educational process whose purpose is to promote the formation of global citizens and critics committed to transform the world into a fairer place and to advocate for Human Rights.
\end{abstract}

\section{Keywords}

Development Education - Citizenship education - Global education - Citizenship.

\section{Introducción}

La educación es un pilar clave para lograr la transformación social, por ello no cabe duda de que la Educación para el Desarrollo (en lo sucesivo, ED) posee una importancia especial a la hora de formar ciudadanos globales que aboguen por un mundo justo y solidario (WAGNER, 2015). Esta relevancia se ve reflejada en los argumentos de autores como Ruiz-Varona y Celorio (2012) quienes afirman que la ED “[...] nunca antes había gozado de tanta consideración como en estos últimos años” (p. 80).

Este estudio se centra en la problemática existente a la hora de establecer una definición de ED, la cual se entiende como un laberinto debido a la posibilidad de comprenderla desde diversos enfoques (político, científico, educativo). Bernal y Carrica (2015), De Castro (2013) y Thomson (2016) afirman que el significado de ED posee un carácter dinámico debido a los cambios que afectan a la sociedad y al concepto de desarrollo; por lo que se han ido provocando modificaciones que han llegado a afectar incluso al nombre que se le ha ido otorgando. A juicio de autores como Baily, 0'Flaherty y Hogan (2017), Celorio y Celorio (2011), o Ortega, Cordón-Pedrosa y Sianes (2013) la 
definición de ED es una cuestión que presenta múltiples connotaciones lo que provoca que sea un asunto que genere reflexiones y debates en el ámbito académico.

En esta línea se enmarca este estudio, el cual pretende analizar 55 definiciones de ED para percibir los rasgos comunes que presentan todas ellas e intentar buscar una definición que pueda aglutinar a todas las demás. Por tanto, la pluralidad y la complejidad que suscita el concepto de ED al no existir una definición única y universalmente aceptada indica el sentido que posee esta investigación, pues busca salir de este laberinto de nociones y sintetizarlas en una única definición sólida y fundamentada que permita encontrar la salida de esta encrucijada tratando de fomentar un acuerdo en la comunidad científica sobre la definición de ED.

Las modificaciones que se han ido produciendo en la definición de la ED se encuentran relacionadas con la evolución histórica, la cual ha afectado directamente a este concepto. Ahora bien, ¿cuál es el recorrido histórico de la ED? La forma más común de estructurar el desarrollo histórico que va desde el origen de la ED en su primera generación hasta la quinta generación en el siglo XX es, en países como España, mediante el modelo generacional propuesto por Mesa (2000a, 2000b). Actualmente, Calvo (2017), Cano (2014) o Sainz de Murieta (2016) afirman que se encuentra en vigor una sexta generación que ampliaría el sistema de los cinco enfoques planteado por autores como: Argibay y Celorio (2005), Boni (2005, 2014), Celorio (2017), u Ortega (2007).

La primera generación tuvo lugar entre los años 40-50 y poseía un carácter crítico $\mathrm{y}$ asistencial. En este enfoque se pretendía informar y conmover a las personas que vivían en los países del Norte transmitiéndoles las desigualdades y los retrasos que sufrían las zonas del Sur (CELORIO; LÓPEZ, 2007). La segunda generación denominada desarrollista, se enmarca en los años 60 y pretende que los países del Norte compartan recursos, tanto económicos como tecnológicos, con las ONG que actúan en los países subdesarrollados (ARGIBAY; CELORIO, 2005).

La tercera generación está marcada por un carácter crítico y solidario que tuvo lugar en los años 70 y 80. En este periodo de tiempo, se empieza a gestar la idea de que los países del Sur se veían afectados por las actuaciones de los países del Norte que acentuaban aún más las desigualdades Norte-Sur (MESA, 2014). Durante los años 80 se desarrolla la cuarta generación centrada en el Desarrollo Humano y el Desarrollo Sostenible. En esta época se amplía el abanico de temas que se tratan en la ED mostrando especial interés en la “[...] interdependencia social, económica, política y ecológica” (BONI, 2005, p. 179); en este sentido, la inclusión de estos nuevos contenidos supone un reto porque resulta necesario que se trabajen nuevos conocimientos de otras educaciones para afines a la ED, entre las que se encuentran la educación para: la paz y el conflicto, el género o el medioambiente. Por tanto, la ED busca una formación global en las personas tratando una amplia variedad de cuestiones relacionadas con la defensa de la justicia social y, a diferencia de la educación para la ciudadanía, no se queda relegada a solamente al desarrollo de personas libres que sepan convivir en sociedad.

La quinta generación denominada para la ciudadanía global surge a mediados los años 90. Aguado (2011) y Fueyo, Hevia y García (2015) advierten que este último enfoque de la EDCG ayuda a fortalecer un modelo de vida social, democrático, justo y participativo y se empeña en lograr una vida feliz para todos los ciudadanos. En los últimos años se 
está gestando la sexta generación de ED que va más allá de la EDCG pues, como afirman Bernal y Carrica (2014), la distinción de este nuevo enfoque se encuentra en pleno debate. Martínez-Usurralde (2013) o Pastoriza (2014) trabajan esta sexta generación de ED desde una visión alternativa que no relega el concepto de ciudadanía global.

Por su parte, Sainz de Murieta (2016) se refiere a esta sexta generación como Educación para la Transformación Social, pues complementa a la generación anterior al resaltar la importancia de que las agendas políticas y los marcos de participación permitan “[...] formar una ciudadanía que, en el actual contexto de globalización, sea capaz de conectar críticamente los problemas locales con los globales y de organizarse para la transformación social” (p. 5). Esta última generación se está gestando actualmente, pero como apunta Calvo (2017) despierta gran interés debido a su estrecha relación con los debates que se pueden encontrar en el contexto internacional (ANDREOTTI, 2014; ANDREOTTI; SOUZA, 2008; BOURN, 2014).

En el ámbito internacional la evolución histórica de la ED está ligada a los discursos económicos y, por este motivo, suele tratarse desde una perspectiva crítica (SELBY; KAGAWA, 2011). Diversas investigaciones relacionan la historia de la ED con el colonialismo (PARMENTER, 2011; CÉSAIRE, 2006), y la descolonización (ANDREOTTI et al., 2015; STEIN; ANDREOTTI; SUSA, 2016) o poscolonialismo (ESCOBAR, 2007; LEETOY, 2016; OXLEY; MORRIS, 2013). Estos paradigmas de la ED se encuentran relacionados con la globalización, el capitalismo y el neoliberalismo, puesto que han tratado de transmitir de la misma forma que el valor de una persona no reside en los objetos que puede producir o poseer; en otras palabras, se ha criticado que "[...] la inmensa mayoría de la humanidad no tiene más que el derecho de ver, oír y callar” (GALEANO, 2000, p. 222).

En la actualidad, autores como Bourn (2014) o Bourn y Brown (2011) se refieren a la ED como Educación para la Ciudadanía Global (EDCG); ambos afirman que la ED deben enseñar diversas cuestiones globales como los Derechos Humanos, la justicia social, las relaciones de interdependencia entre países o el Desarrollo Sostenible. Andreotti (2006, 2014), por su parte, habla de Educación para la Ciudadanía Global Crítica, ya que considera necesario alfabetizar críticamente a las personas para que sean capaces de analizar y reflexionar sobre la realidad social y las situaciones de desigualdad existentes.

Cabe destacar que la manera en la cual la ED ha sido abordada desde el ámbito de la educación formal no ha adquirido la presencia deseada aunque "[...] a pesar de reconocer estas dificultades, se ha mejorado muchísimo el trabajo de los centros educativos" (ARGIBAY; CELORIO, 2005, p. 61). En este aspecto, la ED se ha visto reforzada en el sector formal gracias a las actuaciones llevadas a cabo por diferentes ONGD e incluso, por los propios docentes que han intentado elaborar o incluir en sus programaciones y materiales didácticos los contenidos relativos a la ED (ARGIBAY; CELORIO, 2005).

\section{Objetivos}

El propósito principal de este artículo es analizar el concepto de ED debido a la complejidad que suscita el propio término. En base a este objeto de investigación se plantean los siguientes objetivos: 
- Analizar de forma crítica los documentos encontrados para elaborar un estado de la cuestión que permita vislumbrar las características esenciales de las definiciones de ED existentes hasta la fecha.

- Proponer una definición de ED tomando como base el análisis de las definiciones estudiadas cualitativamente.

\section{Metodología}

La presente investigación emplea una metodología cualitativa para analizar las definiciones de ED seleccionadas. Este análisis de contenido se ha realizado mediante el programa de análisis cualitativo NVivo 10, el cual facilita algunas tareas más mecánicas propias de la investigación debido a las prestaciones que ofrece este software (TRIGUEROS; RIVERA; TORRE, 2011; VALDEMOROS; PONCE; SANZ-ARAZURI, 2011).

Con la finalidad de localizar las diferentes definiciones que se otorgan al concepto de ED se ha efectuado una búsqueda de información principalmente en: PROQUEST, SCOPUS, JSTOR y Web of Science. Se seleccionaron estas bases de datos debido a su prestigio en el ámbito académico, ya que son las que se utilizan habitualmente para la investigación en el área de las Ciencias Sociales. Además, se debe resaltar que la mayoría de los documentos en español han sido seleccionadas debido a su impacto y a su alto índice de citas. Los términos claves empleado en las búsquedas ha sido: Educación para el desarrollo, Education for development y development education, en todos los casos se han incluido comillas para tratar de obtener resultados más precisos.

Tras recopilar diversos documentos que establecen 55 definiciones diferentes que se asocian a lo largo de los años al concepto de ED, se ha procedido a elaborar una base de datos en Excel en la cual se incluye información relativa al autor, el año y su definición.

Una vez elaborada la base de datos, se procedió a importar las definiciones de ED al software de análisis cualitativo NVivo, el cual ha permitido obtener los resultados que se pasan a comentar en el siguiente apartado. Este análisis cualitativo se ha efectuado aplicando tres estrategias básicas que proporciona NVivo: consulta de frecuencia de palabras (se han agrupado los conceptos aplicando la opción de unificar las palabras similares), se ha realizado una nube de palabras para que se puedan apreciar de manera visual los veinte conceptos más empleados en las definiciones analizadas y se han elaborado árboles de palabras únicamente con los términos que requerían un análisis en profundidad (educación, desarrollo y persona) con el propósito de observar las relaciones que se establecen entre ellos.

\section{Resultados}

En un primer acercamiento a la base de datos elaborada, se puede observar en el Gráfico 1 el número de definiciones recopiladas en función de los años de publicación de las referencias consultadas; el año del que más definiciones de ED es 2012. Seguidamente, estarían los años 2004, 2011, 2014, 2017 por lo que se denota la importancia que posee la ED en los últimos años. Se debe puntualizar que la recopilación de estos documentos sobre 
el concepto ED se ha realizado en el primer trimestre del año 2018, por lo que debido a la incapacidad de establecer una definición estándar a medida que pasen los meses este número de definiciones irá aumentando.

Gráfico 1- Años y número de definiciones recopiladas

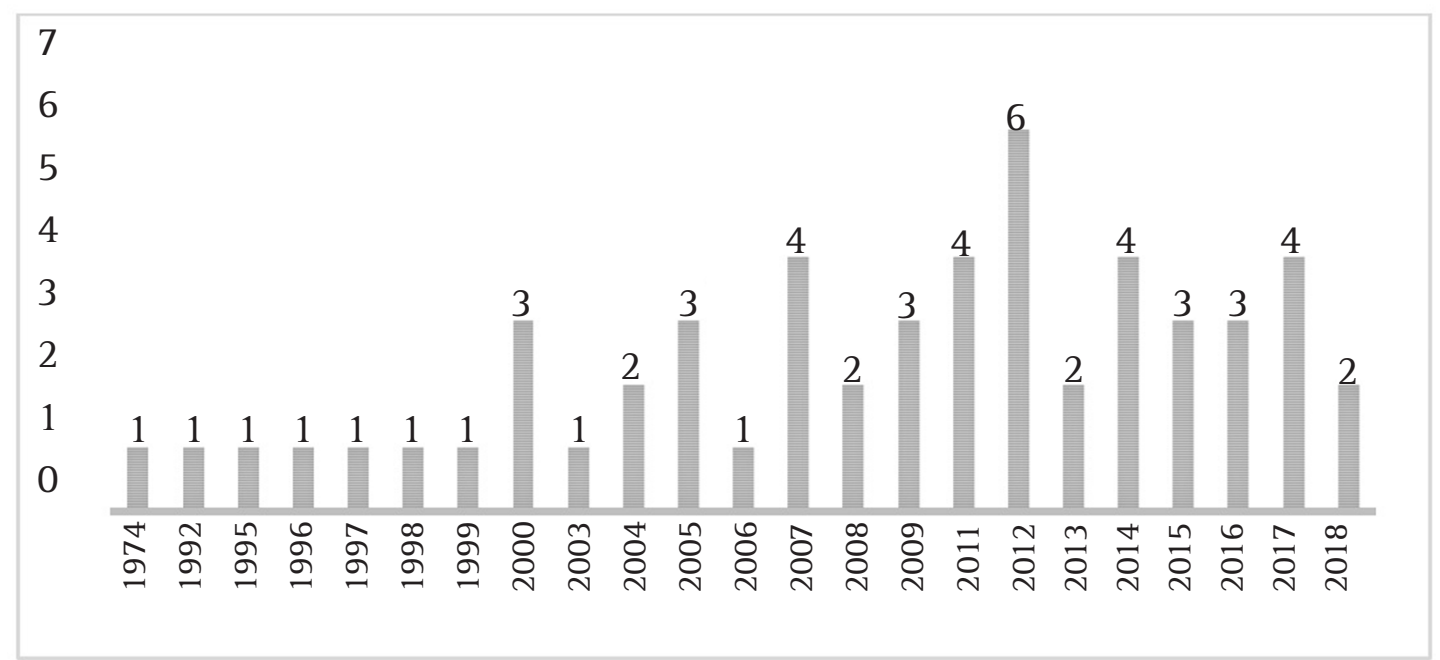

Fuente: Elaboración propia.

El Gráfico 2 agrupa en intervalos las diferentes definiciones estudiadas y, por tanto, refleja la evolución constante en la definición del término ED desde sus inicios hasta la época actual. Como se anticipaba ante los resultados plasmados en el Gráfico 1 los últimos años se aprecia un notable aumento de las definiciones propuestas por los autores en el campo de la ED, lo cual denota que este artículo abarca una temática de interés en el ámbito científico actual a nivel internacional.

Gráfico 2- Definiciones recogidas agrupadas en intervalos

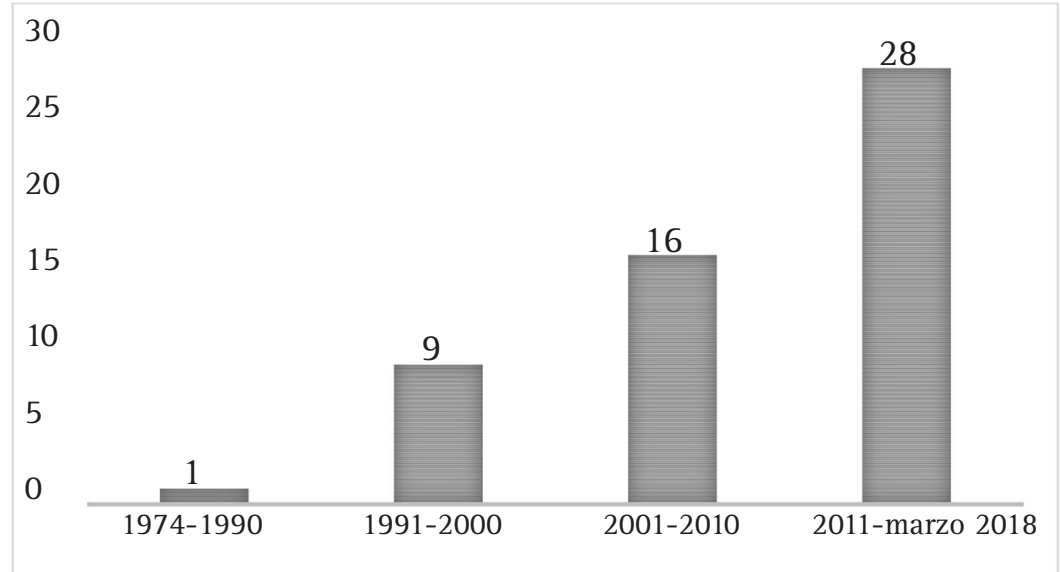

Fuente: Elaboración propia. 
Tras esta primera aproximación a los documentos seleccionados, se importaron las definiciones al software de análisis cualitativo NVivo 10 para poder extraer información sobre los conceptos más relevantes de los significados analizados. La Tabla 1 muestra los veinte conceptos más repetidos entre todas las defıniciones recopiladas en la base de datos.

Tabla 1- Conceptos más repetidos en las definiciones de ED

\begin{tabular}{|c|c|c|c|c|}
\hline Palabra & Longitud & Conteo & Porcentaje ponderado (\%) & Palabras similares \\
\hline proceso & 7 & 39 & 002 & proceso, procesos \\
\hline desarrollo & 10 & 38 & 002 & desarrollo \\
\hline social & 6 & 26 & 001 & social, sociales \\
\hline personas & 8 & 21 & 001 & persona, personas \\
\hline educación & 9 & 20 & 001 & educación, educación \\
\hline humano & 6 & 19 & 001 & humano, humanos \\
\hline educativo & 9 & 19 & 001 & educativo, educativos \\
\hline global & 6 & 16 & 001 & global \\
\hline mundo & 5 & 15 & 001 & mundo \\
\hline sostenible & 10 & 13 & 001 & sostenible \\
\hline conciencia & 10 & 13 & 001 & conciencia, conciencias \\
\hline acción & 6 & 12 & 001 & acción \\
\hline solidaridad & 11 & 12 & 001 & solidaridad \\
\hline valores & 7 & 12 & 001 & valores \\
\hline ciudadanía & 10 & 11 & 001 & ciudadanía \\
\hline comprensión & 11 & 11 & 001 & comprensión \\
\hline comprometida & 12 & 11 & 001 & comprometida, comprometidas \\
\hline norte & 5 & 11 & 001 & norte \\
\hline actitudes & 9 & 10 & 001 & actitudes \\
\hline crítica & 7 & 10 & 001 & crítica, críticas \\
\hline
\end{tabular}

Fuente: Elaboración propia.

El programa NVivo 10 proporciona la posibilidad de agrupar las palabras en función de su semejanza, lo cual ha evitado que se multiplicaran en exceso los conceptos similares. Antes de obtener estos veinte conceptos se tuvo que proceder a eliminar manualmente las palabras vacías o carentes de significado, pues no aportaban ningún tipo de información y eran irrelevantes para esta exploración. Con la finalidad de mostrar de forma visual los veinte conceptos más repetidos en las definiciones, se puede apreciar en la Figura 1 la nube de palabras obtenida. 
Figura 1- Nube con los veinte conceptos más empleados en las definiciones de ED

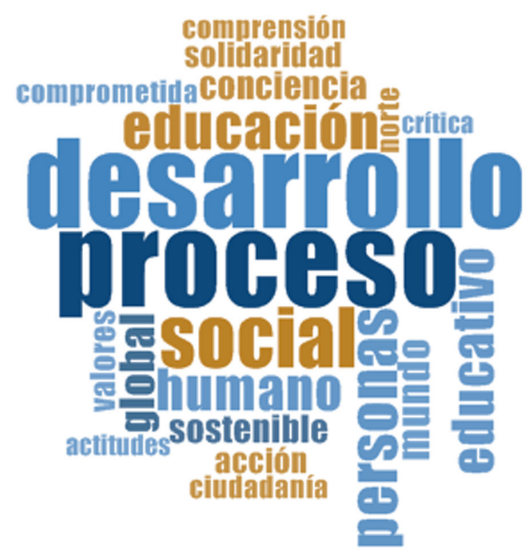

Fuente: Elaboración propia.

El software NVivo 10 ofrece la posibilidad de establecer árboles de palabras que permiten revisar las relaciones que surgen entre conceptos. En este estudio, dado que estamos buscando una aproximación de la definición más idónea para la ED y debido a que este término se compone de dos constructos educación y desarrollo se ha considerado apropiado observar las conexiones existentes entre ambos conceptos.

La Figura 2 muestra el árbol de palabras que emerge del concepto educación. En estas ramificaciones se puede apreciar la presencia de los dos pilares básicos de la educación: las personas en formación y el profesorado. Asimismo, se aprecia la importancia de educar para impulsar un cambio en la sociedad.

Figura 2- Árbol de palabras del concepto educación

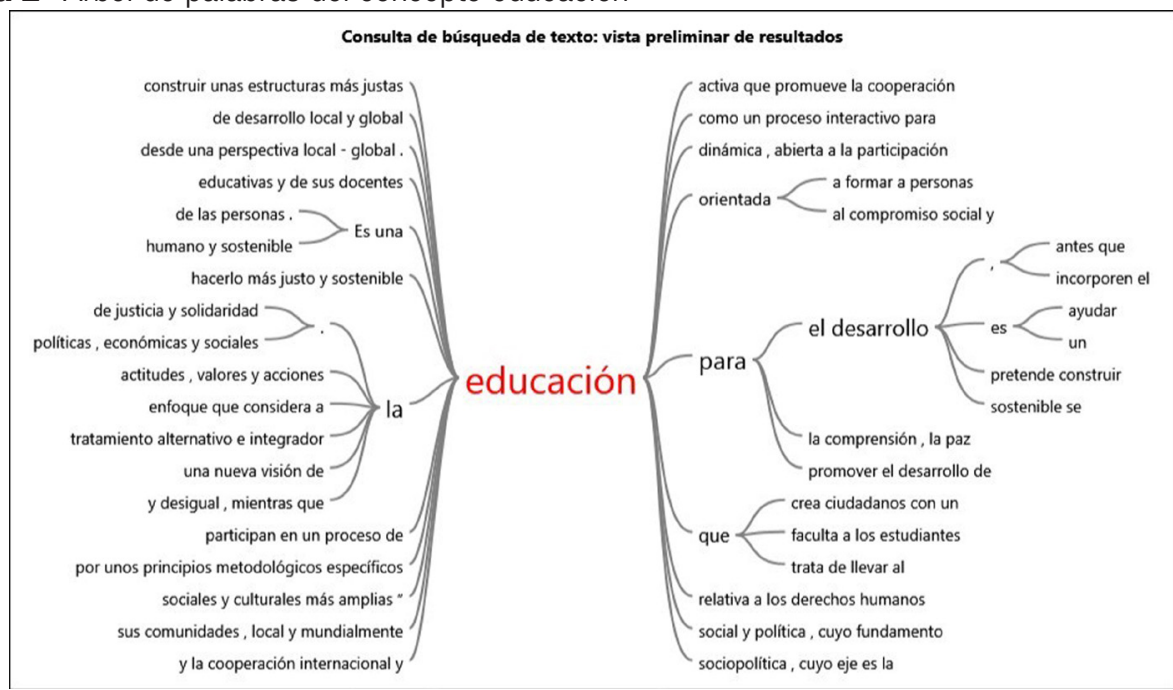

Fuente: Elaboración propia. 
Seguidamente, la Figura 3 recoge el árbol de palabras de las relaciones que surgen del concepto desarrollo, el cual presenta una ramificación mayor que el concepto anterior. Se debe resaltar, en primer lugar, que la rama más relevante es la que relaciona el concepto con el Desarrollo Humano y percibe que la ED se debe comprender como una estrategia de acción socioeducativa que busca fomentar la autonomía de las personas que tiendan a defender un mundo justo y equitativo.

Figura 3- Árbol de palabras del concepto desarrollo

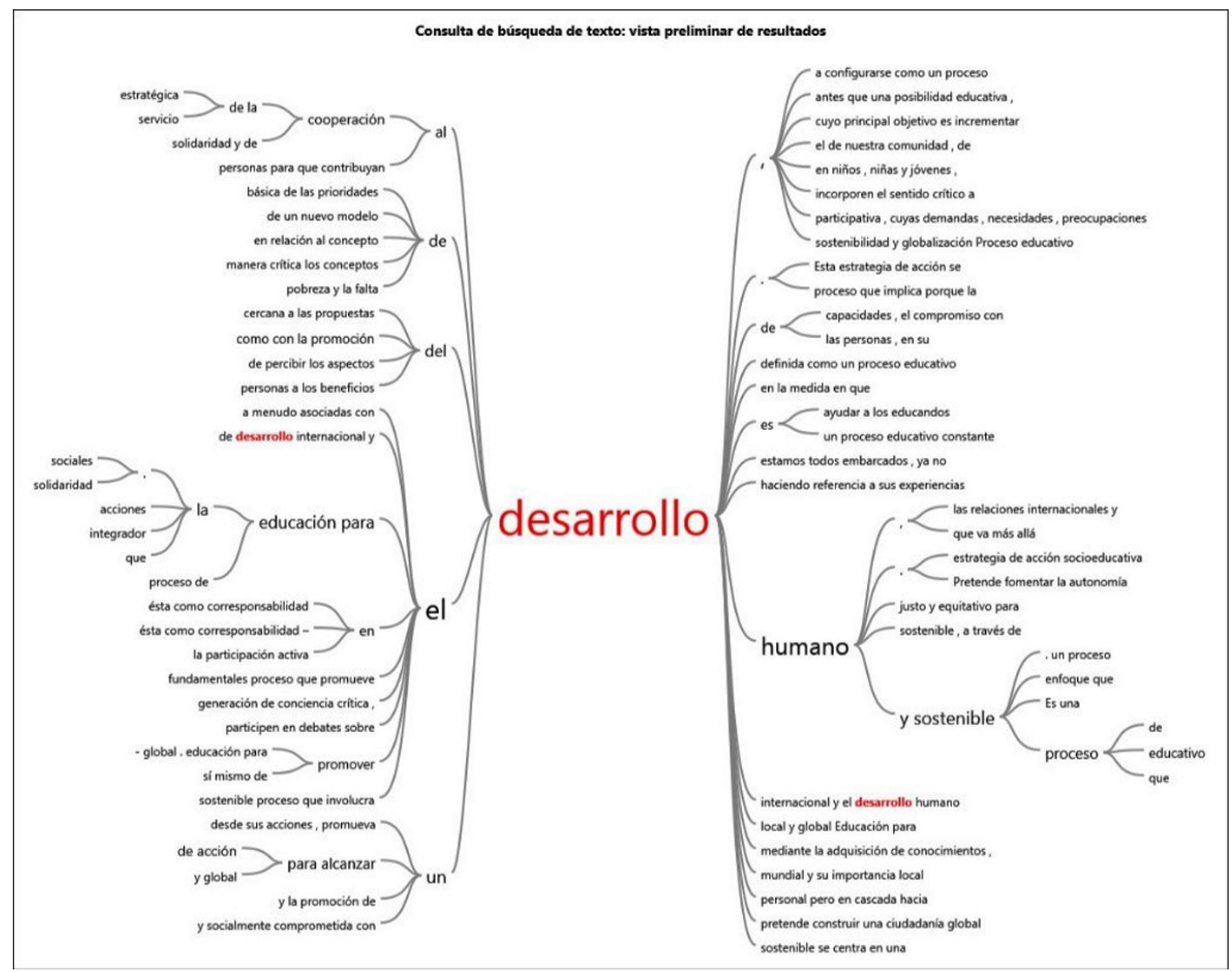

Fuente: Elaboración propia.

Con la intención de completar más profundamente este análisis en la Figura 4 se muestra el árbol de palabras que se asocian dentro de las definiciones examinadas a la palabra persona, pues favorece la visión de estos rasgos y además las personas son un aspecto indiscutiblemente clave dentro de la educación y, especialmente, de la ED. 
Figura 4- Árbol de palabras del concepto personas

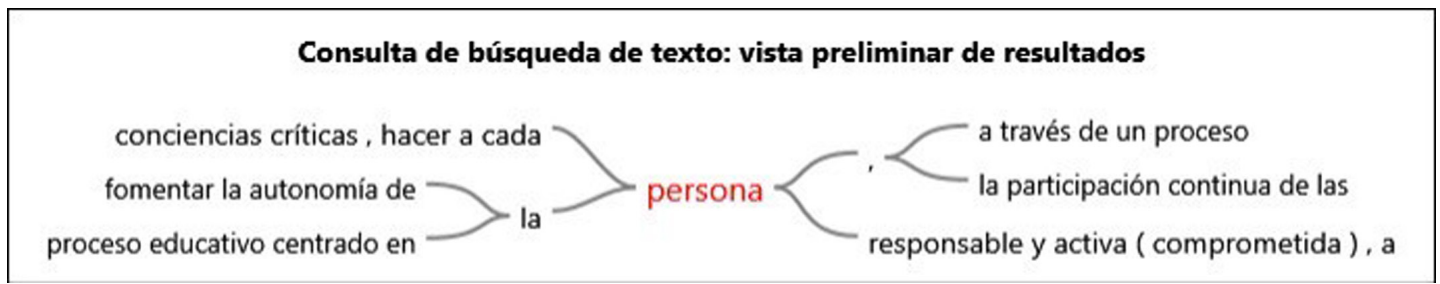

Fuente: Elaboración propia.

Tras realizar este análisis se deben resaltar una serie de rasgos propios que se han ido observando durante el estudio de las definiciones de ED. Concretamente, se recogen en la Tabla 2 una sintesis de las preguntas y las respuestas que se han ido proporcionando en diversos aspectos que se deben contemplar para establecer una adecuada definición de ED.

Tabla 2- Aspectos clave en la definición de ED

\begin{tabular}{|c|c|}
\hline PREGUNTAS & RESPUESTAS A LAS CUESTIONES PLANTEADAS SOBRE ED \\
\hline $\begin{array}{l}\text { ¿Cuáles son las } \\
\text { características de la ED? }\end{array}$ & $\begin{array}{l}\text { La ED debe ser una educación activa, abierta a la participación, que forme personas autónomas comprometidas } \\
\text { con la sociedad y desarrolle las conciencias críticas de los estudiantes. }\end{array}$ \\
\hline $\begin{array}{l}\text { ¿Qué temas se trabajan } \\
\text { desde la ED? }\end{array}$ & $\begin{array}{l}\text { Los DDHH, las interrelaciones existentes entre los países Norte-Sur, las problemáticas relacionadas con el } \\
\text { desarrollo local y global, el DS, el DH, la solidaridad, la justicia social, la equidad, la cooperación y las injusticias. }\end{array}$ \\
\hline $\begin{array}{l}\text { ¿Qué se persigue con } \\
\text { la ED? }\end{array}$ & $\begin{array}{l}\text { La ED pretende despertar en las personas actitudes de solidaridad, autonomía, cooperación, compromiso ante la } \\
\text { problemática que afecta a la sociedad local y al mundo en general y ante las injusticias actuando a favor de la } \\
\text { defensa de los DDHH. }\end{array}$ \\
\hline
\end{tabular}

Fuente: Elaboración propia.

\section{Discusión}

En primer lugar, respecto a las ramificaciones que emergen del concepto educación (véase Figura 2), se puede apreciar que la ED busca construir una sociedad más justa que abogue por el desarrollo local y global. Acorde con esta idea, Sheridan, Landy y Stout (2017) afirman que la ED debe contribuir a la defensa de la justicia social global. Otro aspecto que se aprecia en estas ramificaciones es la importancia de que ED despierte el interés por cooperar e incite a las personas a que participen en la sociedad. En este sentido, Rodríguez y Pérez (2014) se muestran acordes con la idea de fomentar la cooperación al desarrollo para concienciar a las personas de la realidad social. Incluso, Ortega, Cordón-Pedregosa y Sianes (2013) añaden que la ED es un instrumento que se encuentra a disposición de la cooperación al desarrollo.

Cabe añadir que la ED aparece caracterizada en las ramificaciones como un proceso interactivo, abierto y dinámico. Acorde con estos rasgos, Brown (2015) comprende la ED 
como un proceso de aprendizaje activo y, por su parte, Boni (2005) destaca el carácter transformador que posee la ED. Asimismo, haciendo hincapié en la Figura 2 se puede apreciar la importancia de formar personas comprometidas socialmente que promuevan el desarrollo de un mundo más justo, sostenible y humano. Regan (2006) y Solano (2009) establecen que una de las funciones principales de la ED es crear personas comprometidas. Gómez y González (2011) añaden que este compromiso debe poseer carácter social, pues debe ir destinado a conocer las desigualdades existentes entre países y a defender la transformación de la sociedad actual en una más equitativa y justa. O'Flaherty y Liddy (2017) y Ortega (2007) se muestran acordes con la idea anterior, pues afirman que la ED debe promover una ciudadanía solidaria que esté comprometida y actúe a favor de la igualdad impulsando el Desarrollo Humano y Sostenible.

En segundo lugar, en la Figura 3 también se puede observar una ramificación que trata el Desarrollo Sostenible el cual, según se puede interpretar, es entendido como un proceso educativo que debe hacer conscientes a las personas de las problemáticas locales y globales por lo que aboga por construir una ciudadanía global. Monk (2014) afirma que en la época actual resulta complejo ignorar el aumento de la problemática diferenciación existente entre sociedades ricas y pobres por lo que el papel que juega la ED a la hora de formar ciudadanos con conciencias críticas es imprescindible. En esta misma línea, McCloskey (2017) se muestra a favor de que la ED forme personas que sean capaces de reflexionar sobre las cuestiones de desigualdad que atañen al mundo a nivel local y global.

Cabe destacar otro de los aspectos que se considera relevante, en este caso, la asociación del concepto desarrollo con la importancia del perfil de las personas que se deben formar. Se debe destacar que los ciudadanos globales que se deben formar desde la ED deben estar comprometidos con la sociedad; además, se debe generar en ellos una conciencia crítica que les incite a ser solidarios y activos frente a las problemáticas e injusticias presentes en nuestro mundo actual, especialmente la lucha contra la pobreza, la desigualdad o la violación de los Derechos Humanos. Acorde con esta idea, Bentall y Mcgough (2013) y Pashby (2015) comentan que resulta imprescindible avivar "[...] un sentido de ciudadanía global a través de la responsabilidad hacia los demás y respetando los derechos humanos" (PASHBY, 2015, p. 355).

En tercer lugar, en el árbol de palabras de la Figura 4 centrado en las relaciones que surgen del concepto persona, se puede establecer que la ED se entiende como una educación abierta y dinámica que desea fomentar la autonomía de las personas. A juicio de Celorio y López (2007), Fernández y Martínez-Usurralde (2016), o Salter y Halbert (2017), la ED es un proceso educativo que hace hincapié en la formación de una ciudadanía comprometida; es decir, la ED que debe ser capaz de crear personas autónomas y libres que actúen a favor de la igualdad y la justicia social.

Por otra parte, un aspecto muy importante que aparece reflejado en dicho árbol de palabras es la necesidad que tiene la ED de forjar conciencias críticas. Andreotti (2006, 2014), Bamber, Lewin y White (2017) o Bentall y Mcgough (2013) confirman esa idea estableciendo que la ED tiene que enseñar a pensar críticamente a las personas para despertar en ellas un fuerte compromiso, a favor del desarrollo de un mundo más equitativo y defiendan el cumplimiento de los Derechos Humanos. 
Finalmente, tomando referencia los resultados obtenidos en este análisis exploratorio, se puede establecer una definición de ED que recoja la esencia de todas las propuestas analizadas. En este caso, se podría definir la ED como: proceso educativo abierto a la participación que pretende crear personas autónomas que posean conciencias críticas y un fuerte compromiso social. Su finalidad es transmitir la importancia de defender los Derechos Humanos, las interrelaciones existentes entre las sociedades Norte-Sur y cuestiones tan imprescindibles para la sociedad como el Desarrollo Sostenible, el Desarrollo Humano o la justicia social. Es decir, la ED pretende crear ciudadanos globales críticos que actúen a favor de la solidaridad, el respeto, la equidad, la cooperación y la defensa de los Derechos Humanos para transformar el mundo, como diría Freire (1997), en "[...] una sociedad menos desagradable, menos malvada, más humana” (FREIRE, 1997, p. 115).

\section{Conclusiones}

El estudio que se ha plasmado en líneas anteriores ha permitido cumplir los objetivos previstos inicialmente. El propósito principal del estudio se ha cumplido puesto que se ha analizado el concepto de ED utilizando un software cualitativo que ha permitido ir desgranando las características comunes de las definiciones analizadas. Asimismo, a lo largo del artículo se vislumbra que el primer objetivo se ha logrado al ir examinando de forma crítica los diversos documentos que se han localizado en las bases de datos, lo cual ha permitido realizar una discusión sobre las particularidades que presenta el concepto de ED.

Por otra parte, el segundo objetivo se ha alcanzado al tratar de proponer una definición de ED en base a las definiciones estudiadas cualitativamente. En este sentido, se ha comprobado que el término ED presenta controversias y dificultades a la hora de establecer una única definición tanto a nivel internacional. Sin embargo, durante este estudio se ha podido deducir que a la hora de definir la ED se incluyen cuáles son las características, los temas que se trabajan y las finalidades propias de este concepto.

En síntesis, dada la escasez de investigaciones relacionadas con definición de ED debido a su complejidad y su dinamismo, este estudio supone un avance relevante al intentar proporcionar una definición fundamentada en múltiples autores y ampliar los conocimientos existentes sobre esta problemática.

Adicionalmente, la principal limitación de este estudio es la propia dinámica del concepto, pues puede verse sometido a modificaciones con el paso del tiempo debido a las modificaciones que emergerán de los cambios sociales y que afectarán a la búsqueda de nuevas salidas al laberinto de la definición de ED. Esta cuestión presenta líneas futuras innovadoras de investigación como la realización de una consulta a expertos que hayan trabajado cuestiones de ED mediante un estudio Delphi para que aporten su definición de ED y se pueda llegar a un acuerdo de definición según la visión de estos profesionales.

Por consiguiente, se ha llegado a la conclusión de que la ED es un proceso educativo que tiene como finalidad promover una ciudadanía global autónoma y crítica que actúe en defensa de los Derechos Humanos y la justicia social apostando por un mundo más sostenible y justo. 


\section{Referencias}

AGUADO, Guillermo. Educación para el desarrollo y la ciudadanía global. Madrid: Intered, 2011.

ANDREOTTI, Vanessa. Critical and transnational literacies in international development and global citizenship education. Sisyphus Journal of Education, Lisboa, v. 2, n. 3, p. 33-50, 2014.

ANDREOTTI, Vanessa. Soft versus critical global citizenship education. Policy \& Practise, Belfast, n. 3, p. 40-51, 2006.

ANDREOTTI, Vanessa.; SOUZA, Lynn. Global learning in the "knowledge society". ZEP, Frankfurt, n. 31, p. $7-12,2008$.

ANDREOTTI, Vanessa et al. Mapping interpretations of decolonization in the context of higher education. Decolonization, Toronto, v. 4, n. 1, p. 21-40, 2015.

ARGIBAY, Miguel; CELORIO, Gema. La educación para el desarrollo. Vitoria: Gobierno Vasco, 2005.

BAILY, Fiona; O'FLAHERTY, Joanne; HOGAN, Deirdre. Exploring the nature and implications of student teacher engagement with development education initiatives. Irish Educational Studies, Limerick, v. 36, n. 2, p. 185-201, 2017.

BAMBER, Philip; LEWIN, Davies; WHITE, Morgan. (Dis-)locating the transformative dimension of global citizenship education. Journal of Curriculum Studies, Vienna, v. 272, p. 1-27, 2017.

BENTALL, Clare; MCGOUGH, Hannah. Young people' s personal engagement with global learning in further education. International Journal of Development Education and Global Learning, London, v. 5, n. 3, p. 46-67, 2013.

BERNAL, Aurora; CARRICA, Sarah. Mujer, desarrollo y educación para el desarrollo. Edetania, Valencia, n. 46, p. 181-200, 2014.

BERNAL, Aurora; CARRICA, Sarah. Educación para el desarrollo y enseñanza obligatoria. Revista Española de Pedagogía, Logroño, n. 265, p. 499-515, 2015.

BONI, Alejandra. La educación para el desarrollo en la enseñanza universitaria como una estrategia de la cooperación orientada al desarrollo humano. 2005. (Tesis Doctoral) - Universidad de Valencia, Valencia, 2005.

BONI, Alejandra. Un análisis de los discursos institucionales en la cooperación y la educación desde la perspectiva de la educación para la ciudadanía global: reflexiones a partir del caso español. Sinergias, Oporto, n. 1, p. 101-115, 2014.

BOURN, Douglas. The theory and practice of global learning. London: Development Education Research Centre, 2014. 
BOURN, Douglas; BROWN, Kate. Young people and international development: engagement and learning. London: Development Education Research Centre, 2011.

BROWN, Eleanor. Models of transformative learning for social justice: comparative case studies of nonformal development education in Britain and Spain. Compare, London, v. 45, n 1, p. 141-162, 2015. Disponible en: <https://doi.org/10.1080/03057925.2013.841469>. Acceso en: 1 dic. 2018.

CALVO, Adelina. The state of development education in Spain: initiatives, trends and challenges. International Journal of Development Education and Global Learning, London, v. 9, n. 1, p. 18-32, 2017. Disponible en: $<$ https://doi.org/10.18546/IJDEGL.9.1.03>. Acceso en: 1 dic. 2018.

CANO, Ana. Exploración de las prácticas docentes con enfoque de educación para el desarrollo para la ciudadanía global: aproximación diagnóstica en los títulos de grado de las universidades españolas tras la implementación del EEES. 2014. 466 p. Tesis (Doctorado) - Facultad de Ciencias de la Educación, Universidad de Las Palmas de Gran Canaria, Las Palmas de Gran Canaria, 2014.

CELORIO, Gema. Educación crítica y transformadora: marco teórico-pedagógico para integrar la soberanía alimentaria con enfoque de género en los centros de Secundaria. Bilbao: Hegoa, 2017.

CELORIO, Gema; LÓPEZ, Alicia. Diccionario de educación para el desarrollo. Bilbao: Hegoa, 2007.

CELORIO, Juan José; CELORIO, Gema. ¿Educación? para el ¿desarrollo? Pueblos, n. 46, p. 1-3, 2011.

CÉSAIRE, Aimé. Discurso sobre el colonialismo. Madrid: Akal, 2006.

DE CASTRO, Manuel. Dónde y cómo introducir la educación para el desarrollo en los centros educativos. Madrid: CCS, 2013.

ESCOBAR, Arturo. La invención del tercer mundo construcción y deconstrucción del desarrollo. Venezuela: El Perro y la Rana, 2007.

FERNÁNDEZ, Yolanda; MARTÍNEZ-USARRALDE, María. Cuando sentimiento y acción confluyen en la práctica educativa. Alianzas entre educación para el desarrollo y aprendizaje servicio. Ridas, n. 2, p. 111138, 2016. Disponible en: <https://doi.org/10.1344/RIDAS2016.2.6>. Acceso en: 1 dic. 2018.

FREIRE, Paulo. A la sombra de este árbol. Barcelona: El Roure, 1997.

FUEYO, Aquilina; HEVIA, Isabel; GARCÍA, Sara. Haciendo educación para el desarrollo: guía didáctica. Oviedo: Universidad de Oviedo, 2015.

GALEANO, Eduardo. Patas arriba: la escuela del mundo al revés. Madrid: Siglo XXI Editores, 2000.

GÓMEZ, Mercerdes; GONZÁLEZ, Inmaculada. Las universidades ante el reto de la educación para el codesarrollo. Revista Española de Educación Comparada, Madrid, n. 17, p. 87-110, 2011. Disponible en: $<$ https://doi.org/10.5944/REEC.17.2011.7942>. Acceso en: 1 dic. 2018. 
LEETOY, Salvador. Notas sobre modernidad, decolonialidad y agencia cultural en Latinoamérica. Chasqui, Quito, n. 131, p. 47-62, 2016.

MARTÍNEZ-USARRALDE, María. Otras metodologías son posibles... y necesarias: "cuando la cooperación para el desarrollo en educación encontró al APS (aprendizaje servicio)". Valencia: Universidad de Valencia, 2013.

MCCLOSKEY, Stephen. In the age of Brexit and Trump, we need "development education" more than ever. Open Democracy, London, p. 1-4, 2017. Disponible en: <https://www.opendemocracy.net/wfd/stephenmccloskey/in-age-of-brexit-and-trump-we-need-development-education-more-than-ever $>$. Acceso en: 2 dic. 2018.

MESA, Manuela. La educación para el desarrollo: entre la caridad y la ciudadanía global. Papeles de Cuestiones Internacionales, Madrid, n. 70, p. 11-26, 2000a.

MESA, Manuela. La educación para el desarrollo en la comunidad de Madrid: tendencias y estrategias para el siglo XXI. Informe a la Dirección General de Cooperación y Voluntariado. Madrid: Mime0, 2000b.

MESA, Manuela. Precedentes y evolución de la educación para el desarrollo: un modelo de cinco generaciones. Sinergias, Oporto, n. 1, p. 24-56, 2014.

MONK, David. Introducing corporate power to the global education discourse. Policy \& Practice, Belfast, n. 19, p. 33-51, 2014.

O'FLAHERTY, Joanne; LIDDY, Mags. The impact of development education and education for sustainable development interventions: a synthesis of the research. Environmental Education Research, Melbourne, $\mathrm{n}$. 4622, p. 1-19, 2017. Disponible en: <https://doi.org/10.1080/13504622.2017.1392484>. Acceso en: 10 dic. 2018.

ORTEGA, María Luz. Estrategia de educación para el desarrollo de la cooperación española. Madrid: Ministerio de Asuntos Exteriores y de Cooperación, 2007.

ORTEGA, María Luz; CORDÓN-PEDREGOSA, María; SIANES, Antonio. La educación para el desarrollo en el espacio universitario: de la formación en contenidos a la formación integral. Revista Española Del Tercer Sector, Madrid, n. 25, p. 53-78, 2013.

OXLEY, Laura; MORRIS, Paul. Global citizenship: a typology for distinguishing its multiple conceptions. British Journal of Educational Studies, London, v. 61, n. 3, p. 301-325, 2013. Disponible en: <https://doi. org/10.1080/00071005.2013.798393 >. Acceso en: 1 en. 2019.

PARMENTER, Lynne. Power and place in the discourse of global citizenship education. Globalisation, Societies and Education, Bristol, v. 9, n. 3-4, p. 367-80, 2011. Disponible en: <doi:10.1080/14767724.2 011.605322>. Acceso en: 1 en. 2019.

PASHBY, Karen. Conflations, possibilities, and foreclosures: global citizenship education in a multicultural context. Curriculum Inquiry, Toronto, v. 45, n. 4, p. 345-366, 2015. Disponible en: <https://doi.org/10.108 0/03626784.2015.1064304>. Acceso en: 1 en. 2019. 
PASTORIZA, José Luis. Educar para la ciudadanía global: experiencias, herramientas y discursos para el cambio social. Vigo: Fundación Isla Couto, 2014.

REGAN, Colm. Irish aid and development education: describing ... understanding ... challenging. Dublin: Irish Aid Department of Foreign Affairs, 2006.

RODRÍGUEZ, José; PÉREZ, Victoria. Los programas universitarios para mayores como agente de educación para el desarrollo. Búsqueda, Sucre, n. 13, p. 32-40, 2014.

RUIZ-VARONA, José; CELORI0, Gema. Una mirada sobre las miradas: Ios estudios de diagnóstico en educación para el desarrollo. Revista Electrónica Interuniversitaria de Formación Del Profesorado, Murcia, v. 15, n. 2, p. 79-88, 2012.

SAINZ DE MURIETA, Joseba. El papel de las enseñanzas técnicas universitarias en la cooperación universitaria al desarrollo y en la formación de ciudadanía global y transformadora. Ikastorratza, Leioa, v. 17, p. 1-7, 2016.

SALTER, Peta; HALBERT, Kelsey. Constructing the [parochial] global citizen. Globalisation, Societies and Education, Bristol, v. 15, n. 5, p. 694-705, 2017. Disponible en: <https://doi.org/10.1080/14767724.201 6.1264290>. Acceso en: 26 nov. 2018.

SELBY, David; KAGAWA, Fumiyo. Development education and education for sustainable development: are they striking a faustian bargain? Policy \& Practice, Belfast, n. 12, 15-31, 2011.

SHERIDAN, V; LANDY, David; STOUT, Vanessa. The return of the "black babies": how development education affects schoolchildren's attitudes to the majority world - the trócaire 2012 lenten campaign. Race Ethnicity and Education, Birmingham, n. 3324, p. 1-17, 2017. Disponible en: <https://doi.org/10.1080/13613324. 2017.1417829>. Acceso en: 2 dic. 2018.

SOLANO, Rigoberto. Educación para el desarrollo: hacia una reflexión desde sus conceptos y apuestas. Polisemia, Bogotá, n. 8, p. 13-33, 2009.

STEIN, Sharon; ANDREOTTI, Vanessa; SUŠA, Rene. 'Beyond 2015', within the modern/colonial global imaginary? Global development and higher education. Critical Studies in Education, Glasgow, v. 0, n. 0, p. 1-21, 2016.

THOMPSON, Kenneth. Developing education v. education for development. Annals, Aapss, Philadelphia, n. 424, p. 16-28, 2016.

TRIGUEROS, Carmen; RIVERA, Enrique; TORRE, Eduardo. El chat como estrategia para fomentar el aprendizaje cooperativo. Profesorado, Granada, v.1 5, n. 1, p. 195-210, 2011.

VALDEMOROS, María; PONCE, Ana; SANZ-ARAZURI, Eva. Fundamentos en el manejo del NVivo 9 como herramienta al servicio de estudios cualitativos. Contextos educativos, Logroño, n. 14, p. 11-30, 2011.

WAGNER, Tony. Creating innovators: the making of young people who will change the world. New York: Scribner, 2015. 
Recibido en: 19.02.2019

Revisado en: 08.05.2019 Aprobado en: 21.05.2019

Noelia Santamaría-Cárdaba. Máster en Investigación en Ciencias Sociales, graduada en Educación Primaria. Contratada predoctoral FPU en el Departamento de Sociología y Trabajo Social en la Universidad de Valladolid (España). 